cumulative incidences of WLD were higher for boys than girls (boys: 16.5\%; girls: 9.4\%). Subjects with ADHD were more likely to be male, to be white, to have mothers with fewer years of education, and to have younger mothers at birth compared with subjects without ADHD. Perinatal factors were not different among children with and without $\mathrm{ADHD}$ in this cohort.

ADHD was significantly associated with an increased risk of WLD with RD for both boys and girls. However, the magnitude of increased risk for WLD associated with ADHD was significantly greater for girls than for boys (hazard ratio: girls: 9.8; boys: 4.2; $\mathrm{p}<.001$ ). Without RD, boys and girls are at the same risk of having WLD (hazard ratio: girls: 7.4; boys: 8.6 ; $\mathrm{p}=.64$ ). (Yoshimasu $\mathrm{K}$, Barbaresi WJ, Colligan RC, et al. Writtenlanguage disorder among children with and without ADHD in a population-based birth cohort. Pediatrics September 2011;128(3):e605-e612). (Respond: Slavica K Katusic MD, Mayo Clinic, Division of Epidemiology, 200 First St SW, Rochester, MN 55905. Email: katusic.slavica@mayo.edu).

COMMENT. In a Rochester-Olmsted County, MN-based cohort of children, ADHD is associated with an increased risk of WLD for both boys and girls. The risk of WLD with RD associated with ADHD is higher in girls than in boys. In ADHD children having WLD without RD, boys and girls are at the same risk of having WLD. Neuropsychological evaluation of children with ADHD should include tests for written language and reading. The AAP recommends tests for coexisting conditions in children with ADHD. Regrettably, the availability of such evaluations for LD in many US school populations is drastically diminished in recent months, and specific remedial education for $\mathrm{LD}$ and RD in public schools is often wanting.

\title{
OBSESSIVE-COMPULSIVE DISORDER COGNITIVE BEHAVIOR THERAPY
}

Researchers at University of Pennsylvania School of Medicine, Duke University, Brown, Michigan, and University of Massachusetts, Worcester, as members of the Pediatric-OCD Treatment Study II (POTS II) Randomized Controlled Trial, examined effects of augmenting serotonin reuptake inhibitor (SRI) treatment with short-term cognitive behavior therapy (CBT) in 124 pediatric outpatients, 7 to 17 years of age, with a primary diagnosis of OCD. A positive response to treatment was an improvement in a Children's Yale-Brown OCD Scale score by $30 \%$ or more over 12 weeks. Medication management plus 14 concurrent training sessions in CBT (68.6\% responders) was superior to medication only (30\% responders) or medication plus instruction in CBT only (34\% responders). The plus CBT training session group was superior to the other 2 groups ( $\mathrm{P}<.01$ for both). The plus $\mathrm{CBT}$ instruction group was not statistically superior to medication only group $(\mathrm{P}=.72)$. (Franklin ME, Sapyta J, Freeman JB, et al. Cognitive behavior therapy augmentation of pharmacotherapy in pediatric obsessive-compulsive disorder. The Pediatric OCD Treatment Study II (POTS II) randomized controlled trial. JAMA Sept 21, 2011;306(11);1224-1232). (Respond: Martin E Franklin PhD, 3535 Market St, Ste 600, Department of Psychiatry, University of Pennsylvania School of Medicine, Philadelphia, PA 19104. E-mail: marty@mail.med.upenn.edu). 
COMMENT. Instruction in cognitive behavior therapy (CBT) fails to augment benefits of medication management of OCD whereas the addition of CBT training sessions to medication treatment is associated with a significant improvement in response. The authors advocate dissemination of CBT into community settings beyond the academic context so that children affected may benefit from the combined SRI and CBT treatment for OCD. A familial association of OCD, ADHD and Tourette syndromes suggests an overlapping neurobiology (O'Rourke JA et al. Am J Med Genet B Neuropsychiatr Genet 2011;156(5):553-560).

\section{CNS TUMORS}

\section{BIRTH ANOMALIES AND RISK OF CHILDHOOD CNS TUMORS}

Researchers at Departments of Neurology, Pediatrics and Neurosurgery, Stanford University, CA linked 3733 patients aged 0 to 14 years with CNS tumors listed in the California Registry to a California birth certificate. Odds ratios for reported birth defect and history of pregnancy losses were calculated using logistic regression and adjusted for race, maternal age, birth weight, and birth order. Mothers with - or $>2$ fetal losses after 20 weeks' gestation had a 3-fold risk of offspring with CNS tumors and a 14-fold risk of high-grade glioma. Children with congenital birth defects had an increased risk of the CNS cancers medulloblastoma, primitive neuroectodermal tumor and germ cell tumors, tumors that tend to arise in the midline. Among tumor types in case patients, gliomas were most frequent (57\%): 1380 cases were low-grade gliomas and 757 were high-grade. Embryonal (889 [24\%]) and ependymoma (292 [8\%]) were less frequent. Choroid plexus tumors $(2 \%)$ and craniopharyngiomas $(0.5 \%)$ were rare. Previous pregnancy losses and birth defects may be surrogate markers for gene defects in developmental pathways that lead to CNS tumorigenesis. (Partap S, MacLean J, Von Behren J, Reynolds P, Fisher PG. Birth anomalies and obstetric history as risks for childhood tumors of the central nervous system. Pediatrics Sept 2011;128:e652-e657). (Respond: Sonia Partap MD MS, Division of Child Neurology, Stanford University, 750 Welch Rd, Suite 317, Palo Alto, CA 94304. E-mail: spartap@ stanford.edu).

CGMIMEINT. In this population-based case-control study, mothers who have already lost - or $>2$ fetuses after 20 weeks' gestation have a significant risk of CNS tumors in their offspring, and children with birth defects are also at increased risk, especially of midline CNS tumors.

\section{SLEEP DISORDERS}

\section{NARCOLEPSY AND 2009 H1N1 PANDEMIC IN CHINA}

Onset of narcolepsy in China (1998-2010) is seasonal, with a 6.7-fold increase from November (least frequent) to April (peak frequency). A 3-fold increase followed the $2009 \mathrm{H} 1 \mathrm{~N} 1$ winter influenza pandemic, and this correlation was independent of vaccination. (Han F, Lin L, Warby SC, et al. Ann Neurol 2011;70:410-417). (Respond: Dr Han. E-mail: hanfang1@ @otmail.com; or Dr Mignot: mignot@stanford.edu). 